\title{
Rheological properties of cellulose nanocrystal-embedded polymer composites: a review
}

\begin{abstract}
Nanotechnology provides useful insights into the behavioural properties of materials from the nanoscale point of view, enabling researchers to develop new materials that were previously inconceivable. Cellulose is an ideal candidate for nanomaterial for nanotechnology because of its nanofibrillar structure, abundance, renewability, biodegradability and eco-friendly nature. Nanocrystalline cellulose materials have become the focus many studies related to these materials and their applications. This review summarises the current knowledge on the field of nanomaterials, focussing mainly on the rheological behaviour of polymer nanocomposites embedded with nanocrystalline cellulose. This review will enable better understanding of the use of nanocrystalline cellulose for the development and applications of cellulose nanocrystal-based nanocomposites.
\end{abstract}

Keyword: Cellulose nanocrystals (CNCs); CNC-matrix composites; Rheological properties 